Hydrol. Earth Syst. Sci., 18, 1213-1223, 2014

www.hydrol-earth-syst-sci.net/18/1213/2014/

doi:10.5194/hess-18-1213-2014

(C) Author(s) 2014. CC Attribution 3.0 License.

\title{
Eco-environmentally friendly operational regulation: an effective strategy to diminish the TDG supersaturation of reservoirs
}

\author{
J. Feng, R. Li, R. Liang, and X. Shen \\ Yihuang Road, Chengdu, 610065, China \\ Correspondence to: R. Li (liran@scu.edu.cn) \\ Received: 17 October 2013 - Published in Hydrol. Earth Syst. Sci. Discuss.: 22 November 2013 \\ Revised: 8 February 2014 - Accepted: 12 February 2014 - Published: 28 March 2014
}

State Key Laboratory of Hydraulics and Mountain River Engineering, Sichuan University, No. 24 of South Section One,

\begin{abstract}
The presence of elevated total dissolved gas (TDG) downstream of a spillway may result in an increased incidence of gas bubble disease in fish. Supersaturated TDG is transported and dissipated more slowly in reservoirs than in natural rivers because of a higher water depth and lower turbulence and thus endangers fish. This paper presents the operational regulations concerning the mitigation of the TDG supersaturation impact on fish in the Bala Reservoir on the Zumuzu River. The paper includes the joint operations of the upstream and downstream power stations. A laterally averaged two-dimensional model is employed to simulate the TDG transportation and dissipation in the Bala Reservoir in addition to the hydrodynamics and water temperature. According to a comparison of the simulation results of different operational scenarios, this paper suggests a regulation scheme. With respect to the upstream power station, the adoption of an interval-discharge pattern instead of a continuous-discharge pattern is recommended to minimize the negative effect on the reservoir caused by supersaturated TDG. With respect to the downstream power station, the adoption of a surface tunnel rather than a bottom tunnel is recommended as a release structure. This paper is a reference for the eco-environmentally friendly operation of large and deep reservoirs.
\end{abstract}

\section{Introduction}

Involuntary spillage occurs in the high-runoff season. The phenomenon of TDG supersaturation is observed downstream of a dam spill, which may lead to an increase in the incidence of gas bubble disease in fish (Weitkamp et al., 1980,
2003). Levels of TDG saturation in excess of $130 \%$ have been observed downstream of many high-dam spills (Qu et al., 2011). Supersaturated TDG always dissipates slowly in downstream flood transportation, and the dissipation may continue hundreds of kilometers downstream of the spillway. The dissipation rate depends on factors such as the water depth, turbulence, and temperature (Feng et al., 2010). With the development of continuous cascade hydroelectric power systems, one deleterious impact on TDG transportation and dissipation is that the dissipation rate of TDG decreases substantially with an increase in water depth and a decrease in turbulence when a natural river is transformed into a reservoir. Consequently, the injury to fish is enhanced. The TDG transportation and distribution in a reservoir is closely related to the operation of upstream and downstream dams. To mitigate the detrimental effects to fish in the discharging period and to manage the conflict between the spill discharge and the aquatic system, operational regulation with respect to dam discharge is a viable strategy.

\subsection{The previous studies}

The development of hydroelectric power, especially with the development of continuous cascade systems, and the negative impacts of hydropower stations on the aquatic environment has attracted extensive attention. Recently, ecoenvironmentally friendly operational regulation has been investigated to assuage the conflict between hydropower development and environmental protection. Yang et al. (2013) have proposed an available operational method to decelerate the blooms in the tributary bays of the Three Gorges Reservoir. Lindim et al. (2011) discussed the water quality changes in a large reservoir when water temperature is 
in stratification. An operational proposal is provided that minimizes the negative impact. van Valit (2012) studied the effect of reservoir regulations on stream flow and thermal pollution on the Columbia River. Because climate changes and temperatures have increased in the Mekong Basin in response to hydropower development, Lauri et al. (2012) studied the optimization approach for multiple reservoir operations based on the hydrology analysis for the next 20 to 30 years. Nikoo et al. (2013) developed an integrated water quantity-quality model for optimal water allocation in the reservoir-river basin that considered water supply and quality as targets in addition to hydrologic, water quality, and water demand uncertainties. To explore the impact on habitat suitability of carp in the Yangtze River following the construction of the Gezhouba and the Three Gorges dams, a habitat suitability index model that considered the spawning characteristics of the four main carp species was established and employed by Yi et al. (2010). Cai et al. (2009, 2010) identified optimal strategies in the planning of energy and flood management aiming at fish protection and pursuing eco-environmentally friendly operations. Based on the calculated results, a minimum instream flow and suitable daily discharge increase during the reproduction season is suggested for the protection of the carp species. To balance human and ecosystem needs, Yin et al. (2010) proposed a reservoir operation method that combines reservoir operating rule curves with the regulated minimum water release policy to meet the environmental flow requirements of riverine ecosystems. This operational method is applied to the Tanghe Reservoir and Tanghe River basin. The prior research with respect to eco-environmentally friendly operations for reservoirs focuses on hydrology, sedimentation, water temperature and traditional water quality factors. The operational regulations for mitigating the negative impact of TDG, an important factor with respect to fish, are merely reported. According to the numerical results, Politano et al. (2012) compared the TDG levels at the stilling basin downstream of Wells Dam under different configurations.

Dissolved oxygen (DO) was chosen as the main variable in the early studies on the negative impact on fish caused by dam spilling. Afterwards, TDG is studied as a new variable. According to the investigation of Ma et al. (2013), it is difficult to find a stable relationship between DO and TDG. In the authors' previous studies (Li et al., 2013), it was found that the TDG dissipation process is quantitatively different from the reaeration process of DO. Some biological studies also indicate that the supersaturated TDG is more harmful to the fishes than the supersaturated DO (Rucker, 1976). For these reasons, TDG is chosen as the key variable to study the eco-environmental regulations for mitigating the conflict between dam spilling and fish protection in the paper. The US Environmental Protection Agency has recognized the threat to fish and has set water quality standards for dissolved gas levels at $110 \%$ of saturation (US EPA, 1986).
Prior studies reveal that the injury to fish is enhanced as the supersaturated TDG level and exposure time increases (Bentley et al., 1976; Huang et al., 2010; Chen et al., 2012). The endurance of fish with respect to supersaturated TDG is different depending on the growth period for the fish species (Liang et al., 2013). The impact of interval exposure to supersaturated TDG on fish was examined in a laboratory by Liu et al. (2011). The results demonstrated that the damaged biological function of rock carp as a result of exposure to the TDG supersaturated water can recover when the rock carp is returned to fresh water. The research findings imply that the TDG impact on fish can be mitigated by reducing the exposure time and adjusting the manner of exposure of fish to TDG supersaturation.

The research findings suggest that optimized operational regulation of reservoirs is an effective approach to minimizing TDG levels in the reservoir and to the mitigation of the conflict between dam spilling and the protection of fish

\subsection{Objective}

This paper focuses on an operational strategy to minimize the negative effect of TDG in reservoirs. Numerical simulations are employed to compare the TDG distribution in Bala Reservoir under various configurations. An optimized regulation policy for Bala Reservoir is proposed based on the numerical results. Politano (2009), Urban et al. (2008), and Fu et al. (2010) have developed unsteady two-phase threedimensional models to calculate the TDG downstream evolution of spillways. The accuracy is satisfied in their simulations. However, these models are not applicable to largescale bodies of water, such as reservoirs with a depth of more than $100 \mathrm{~m}$ and more than $100 \mathrm{~km}$ in length because they are limited by time cost and convergence. Therefore, this paper adopts a laterally averaged two-dimensional model that is proposed by the author (Feng et al., 2013) to predict the dynamics and the TDG levels in the simulations. The operational regulations of both the upstream and the downstream power stations are discussed.

\section{The case study}

Bala power station, with a maximum height of $138 \mathrm{~m}$, is a high-dam hydropower station that is to be constructed on the Zumuzu River, source of the Daduhe River. The main discharge structures are a surface spillway tunnel and a bottom spillway tunnel. The elevation of the weir crest of the surface spillway tunnel is 2903 and $2845 \mathrm{~m}$ for the bottom spillway tunnel. The backwater length of Bala Reservoir under normal water level is $26 \mathrm{~km}$. At the upstream end of Bala Reservoir, a controlled cascade, Xiaerga Dam, is in the planning stages. The maximum height is $242 \mathrm{~m}$. The main discharge structure is a spillway. The dissipater of the spillway is designed as a ski-jump type model. 


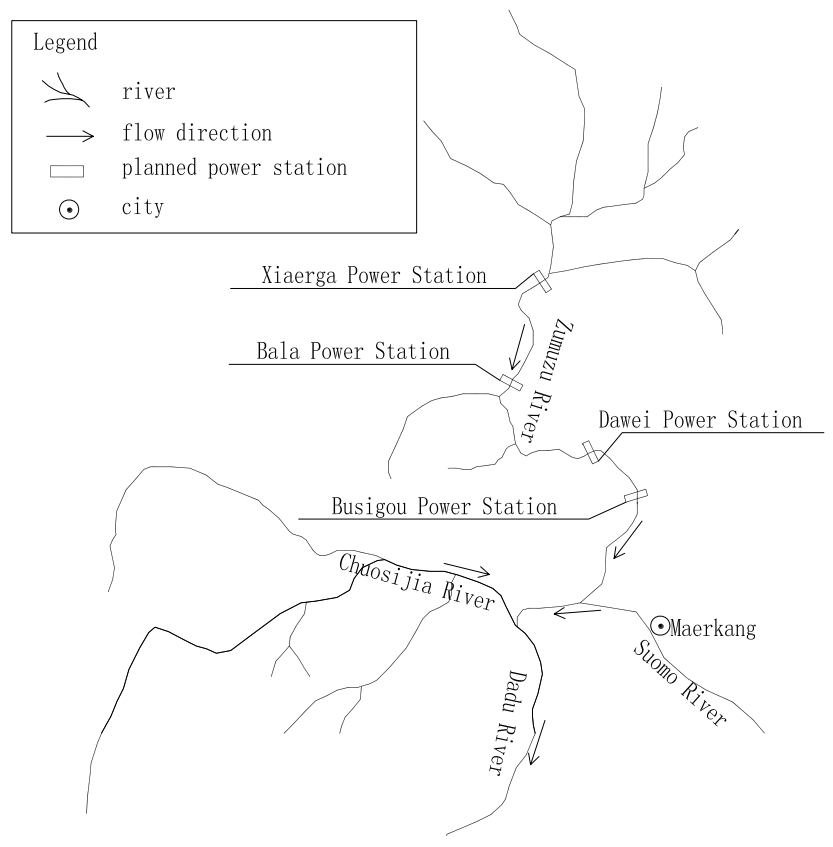

Fig. 1. The cascades on Zumuzu River.

The fish species in the Zumuzu River are rare and endemic. The species include one type of Class II nationally protected Chinese fish, Hucho bleekeri; two protected fish from the Sichuan Province, Schizothorax (Racoma) davidi and Euchiloglanisdavidi; and several endemic fish from the upper reaches of the Yangtze River, Schizothorax (schizothorax) prenanti (Tchang) and Sp. malacanthus chengi. The breeding periods of the rare and protected fishes in Zumuzu River are concentrated from April to July. Thus in the concentrated dam-spilling period, the breeding and the growth of fish will be influenced.

The protection of the ecosystem during the hydropower development is a primary concern for the developers and scientists.

\section{The methods and models}

The TDG transportation and dissipation process is simulated in this study by employing the model developed by Feng et al. (2013). This model is a two-dimensional laterally averaged hydrodynamic and water quality model. The hydrodynamics and the temperature are coupled-solved with the code CE-QUAL-W2. The simulation of the TDG transportation and dissipation is programmed by the authors and incorporated into the CE-QUAL-W2 codes.

\subsection{The governing equations}

The governing equations of the model are listed as follows.

The continuity equation:

$\frac{\partial U B}{\partial x}+\frac{\partial W B}{\partial z}=q B$

The momentum equation:

$\frac{\partial U B}{\partial t}+\frac{\partial U U B}{\partial x}+\frac{\partial W U B}{\partial z}=g B \sin \alpha$

$-\frac{B}{\rho} \frac{\partial p}{\partial x}+\frac{1}{\rho} \frac{\partial B \tau_{x x}}{\partial x}+\frac{1}{\rho} \frac{\partial B \tau_{x z}}{\partial z}$

$\frac{1}{\rho} \frac{\partial p}{\partial z}=g \cos \alpha$

The free water-surface equation:

$B_{\eta} \frac{\partial \eta}{\partial t}=\frac{\partial}{\partial x} \int_{\eta}^{h} U B \mathrm{~d} z-\int_{\eta}^{h} q B \mathrm{~d} z$

The density is essential for the solution of the momentum equations. The equation of state relates the density to the temperature. The following relationship is used in the model:

$$
\begin{aligned}
& \rho=999.845259+6.793952 \times 10^{-2} T_{\mathrm{w}} \\
& -9.19529 \times 10^{-3} T_{\mathrm{w}}^{2}+1.001685 \times 10^{-4} T_{\mathrm{w}}^{3} \\
& -1.120083 \times 10^{-6} T_{\mathrm{w}}^{4}+6.536332 \times 10^{-9} T_{\mathrm{w}}^{5},
\end{aligned}
$$

where $U$ and $W$ denote temporal mean velocity components in a horizontal and vertical direction; $x$ and $z$ represent the horizontal distance and vertical elevation; $B$ is the width; $q$ is the specific discharge; $t$ denotes the time; $g$ is the acceleration of gravity; $p$ represents pressure and $\rho$ denotes the density; $\tau_{x x}$ and $\tau_{x z}$ are defined as the turbulent shear stress acting in $x$ direction on the $x$ face of control volume; $\alpha$ is the angle of the river bed to $x$ direction; $\eta$ and $h$ are the water surface and water depth; and $T_{\mathrm{w}}$ represents the water temperature. The universal transportation equation for scalar variables, such as temperature and TDG, is

$$
\begin{aligned}
& \frac{\partial(\Phi B)}{\partial t}+\frac{\partial(U B \Phi)}{\partial x}+\frac{\partial(W B \Phi)}{\partial z} \\
& =\frac{\partial\left(B D_{x}\right)}{\partial x^{2}}+\frac{\partial\left(B D_{z} \Phi\right)}{\partial z^{2}}+S_{\Phi} B,
\end{aligned}
$$

where $\Phi$ denotes a scalar variable.

The source term for temperature consists only of surface heat exchange:

$$
\begin{aligned}
& H_{\mathrm{n}}=H_{\mathrm{s}}+H_{\mathrm{a}}+H_{\mathrm{e}}+H_{\mathrm{c}} \\
& -\left(H_{\mathrm{sr}}+H_{\mathrm{ar}}+H_{\mathrm{br}}\right),
\end{aligned}
$$

where $H_{\mathrm{n}}$ is the net rate of heat exchange across the water surface; $H_{\mathrm{S}}$ denotes the incident shortwave solar radiation; $H_{\mathrm{a}}$ represents the incident longwave radiation; $H_{\mathrm{sr}}$ and $H_{\mathrm{ar}}$ are the reflected radiations of short waves and long waves, respectively; $H_{\mathrm{br}}$ is the back radiation from the water surface; $H_{\mathrm{e}}$ represents the evaporative heat loss; and $H_{\mathrm{c}}$ denotes heat conduction. 
The source term of TDG is defined as

$S_{\Phi}=k_{T}\left(C_{\mathrm{eq}}-C\right)+\left(K_{\mathrm{L}} a\right)_{\mathrm{s}}\left(C_{\mathrm{s}}-C\right)$,

where $C$ denotes the average TDG concentration expressed as a percent of the saturation concentration, $C_{\mathrm{S}}$ is the saturation concentration at local atmospheric pressure, $C_{\mathrm{eq}}$ is defined as the equilibrium saturation level of each cell relative to the local pressure and temperature, $k_{T}$ is the dissipation coefficient of the water body and is zero when $C_{\mathrm{eq}}-C>0$, $\left(K_{\mathrm{L}} a\right)_{\mathrm{S}}$ is the surface mass transfer coefficient across the airwater surface interface and $a$ denotes the specific surface area.

The following expression is widely used in the evaluation of the surface mass transfer coefficient across the air-water surface interface $K_{\mathrm{L}}$ :

$K_{\mathrm{L}}=0.4 S c^{-1 / 2}(\nu \varepsilon)^{1 / 4}$,

where $S c$ is the Schmidt number $S c=D / \nu, D$ represents the molecular diffusion and $v$ denotes the eddy viscosity of water; $\varepsilon$ is the rate of energy dissipation.

Because the rate of energy dissipation $\varepsilon$ is not calculated in the model, another empirical formula to evaluate the surface mass transfer coefficient, $\left(K_{\mathrm{L}} a\right)_{\mathrm{s}}$, raised by O'Connor (1983), is employed in the model.

$$
\begin{aligned}
& \left(K_{\mathrm{L}} a\right)_{\mathrm{S}}=-0.0045 v_{\mathrm{w}}^{3}+0.1535 v_{\mathrm{w}}^{2} \\
& -0.5026 v_{\mathrm{w}}+0.6885,
\end{aligned}
$$

where $v_{\mathrm{W}}$ represents the wind speed at a height of $10 \mathrm{~m}$.

\subsection{The discrete method}

The finite difference method is implied in the discrete method of governing equations and transportation equation.

The discretization of the free water-surface equation employs an explicit scheme. The discretization of the momentum equation employs a combining method, vertical diffusion is fully implicit and advection uses a time-weighted, central difference, implicit scheme. A unique feature of vertical advection, in the explicit part of the time-weighted scheme is QUICKEST, developed by Leonard, which increases the overall accuracy. The discretization of the transportation equation uses a similar method as the momentum equation.

\subsection{The model calibration and validation}

Field observations were performed between 26 July and 1 August 2008, to study the TDG dissipation in the Dachaoshan Reservoir. Four transects were set along the reservoir to measure the TDG saturation of the water surface. A vertical line was planned at the $500 \mathrm{~m}$ upstream section of the Dachaoshan Dam. The TDG and depth data were collected at depth increments of approximately $5 \mathrm{~m}$.

The numerical model used in this study was employed by the author (Feng et al., 2013) to simulate the unsteady

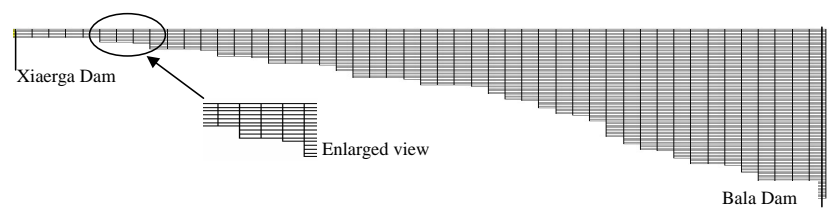

Fig. 2. Grid structure of Bala Reservoir.

dynamics and the TDG in the Dachaoshan Reservoir. The simulation results were consistent with the field measurements collected in this study, including the unsteady TDG saturation at each transect and the vertical distribution at the $500 \mathrm{~m}$ upstream section of the Dachaoshan Dam. The results demonstrated that the laterally averaged two-dimensional model is applicable to the prediction of TDG transportation and dissipation in a reservoir.

The dissipation coefficient of $0.03 \mathrm{~h}^{-1}$ in the Dachaoshan Reservoir is numerically evaluated.

\section{The simulation conditions}

\subsection{The case description and grid generation}

$P=5 \%$ (once in twenty years) is taken as the flow condition at the dam site of Xiaerga, which is equal to $1910 \mathrm{~m}^{3} \mathrm{~s}^{-1}$. The simulated period is $48 \mathrm{~h}$, from 12 August, 00:00 GTM+8 to 13 August, 24:00 GTM+8. The simulated region is $26 \mathrm{~km}$ long and stretches from the Xiaerga Dam to the Bala Dam. The topographic data of the Bala Reservoir are used to build the simulated zone. A structured mesh system, $500 \mathrm{~m}$ in the longitudinal direction and $1 \mathrm{~m}$ in the vertical direction, is generated. The grids of the simulation domain are shown in Fig. 2.

Three scenarios are assumed for simulation according to the operational condition of Bala Reservoir in this paper. First, considering the effect of the discharging frequency, two scenarios are performed, Case 1 and Case 2. With respect to Case $1,12 \mathrm{~h}$ discharging is continuous. With respect to Case 2, the discharging time is equally divided into four time intervals, each lasting $3 \mathrm{~h}$. Second, to compare the effect of outlet elevation on the TDG distribution in the reservoir, Case 3 is set to contrast to Case 1 . In Case 1, the flood runs out through the surface spilling tunnel of Bala at an elevation of $2903 \mathrm{~m}$. In Case 3, the flood runs out through the bottom discharge tunnel at an elevation of $2845 \mathrm{~m}$. The contrasts of the three cases are listed in Table 1.

\subsection{The initial conditions}

The dynamics for this model are computed coupled with the temperature. The initial temperature field is significant to the formal simulation. The heat transfer from the air, the shortwave solar radiation, the incident longwave radiation, the reflected radiations, and the evaporative heat loss are 
Table 1. Case description and boundary conditions.

\begin{tabular}{|c|c|c|c|c|c|c|c|c|}
\hline \multirow{2}{*}{ Case No. } & \multicolumn{5}{|c|}{ Upstream inflow } & \multicolumn{3}{|c|}{ Downstream outflow } \\
\hline & $\begin{array}{l}\text { Discharge } \\
\text { structure }\end{array}$ & $\begin{array}{l}\text { Discharge } \\
\text { pattern }\end{array}$ & $\begin{array}{c}\text { Discharge } \\
\text { flow rate } \\
\left(\mathrm{m}^{3} \mathrm{~s}^{-1}\right)\end{array}$ & $\begin{array}{c}\text { Power flow } \\
\left(\mathrm{m}^{3} \mathrm{~s}^{-1}\right)\end{array}$ & $\begin{array}{c}\text { TDG } \\
\text { saturation } \\
\text { level }(\%)\end{array}$ & $\begin{array}{l}\text { Discharge } \\
\text { structure }\end{array}$ & $\begin{array}{l}\text { Discharge } \\
\text { pattern }\end{array}$ & $\begin{array}{c}\text { Outlet } \\
\text { elevation }(\mathrm{m})\end{array}$ \\
\hline Case 1 & Spillway & Continuous & 1710 & 200 & 141 & Surface spillway tunnel & Continuous & 2903 \\
\hline Case 2 & Spillway & Interval & 1710 & 200 & 141 & Surface spillway tunnel & Interval & 2903 \\
\hline Case 3 & Spillway & Continuous & 1710 & 200 & 141 & Bottom discharge tunnel & Continuous & 2845 \\
\hline
\end{tabular}

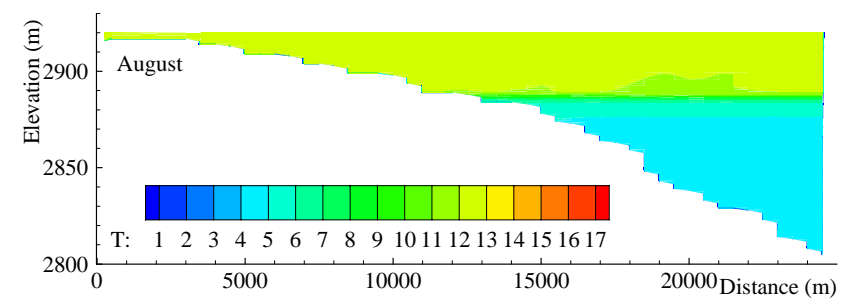

Fig. 3. The initial temperature field of Bala Reservoir $\left(^{\circ}\right)$.

incorporated in the heat transport equation. By means of numerical simulation with a laterally averaged 2-D model, the initial temperature field is pre-computed by circularly calculating the dynamics and the water temperature month by month for 2 years. The resulting temperature field for August is used as the initial field. During the pre-computation, the monthly hydrological data and inflow water temperatures are obtained from the Zumuzu hydrological station. The meteorological data are obtained from the Maerkang meteorological station. Figure 3 presents the initial temperature field for the simulation.

\subsection{The boundary conditions}

The TDG concentration and temperature of the inflow are based on field observation data. The boundary conditions versus time are presented in Figs. 4 and 5.

The power flow is $200 \mathrm{~m}^{3} \mathrm{~s}^{-1}$ and the upstream spillway discharge rate is $1710 \mathrm{~m}^{3} \mathrm{~s}^{-1}$ at the Xiaerga power station. Ignoring the daily regulation of Bala Reservoir, the downstream outflow of Bala Reservoir is assumed to be the same as the upstream inflow and is presented in Figs. 4 and 5.

The TDG supersaturation level downstream of the Xiaerga spillway discharge is calculated with the empirical model proposed by Li et al. (2009). The TDG supersaturation level of power flow is assumed to be the equilibrium saturation of tailrace, $100 \%$. The flow-weighted average method is used to estimate the mixing TDG concentration of spill discharge and power flow. The flow-weighted average TDG is calculated to be $141 \%$. The inflow boundaries of TDG are presented in Figs. 4 and 5.

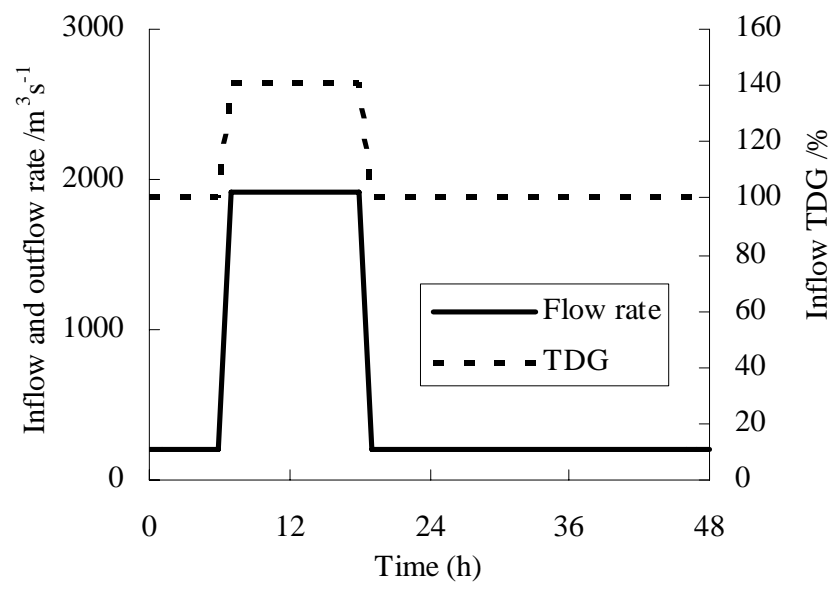

Fig. 4. The flow and TDG boundary conditions for Case 1 and Case 3.

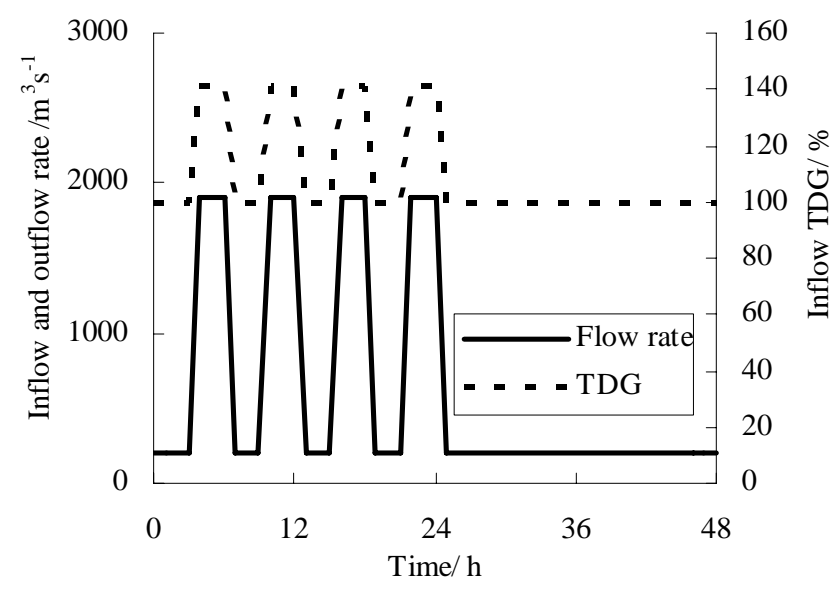

Fig. 5. The flow and TDG boundary conditions for Case 2 .

\subsection{Parameter determination}

The equilibrium saturation concentration $C_{\mathrm{eq}}$ varies with pressure, temperature and turbulence. In this simulation, the effect of hydrostatic pressure on the value of $C_{\text {eq }}$ is considered as the following:

$C_{\text {eq }}=100+10 h$,

where $h$ represents the local depth under the free surface. 


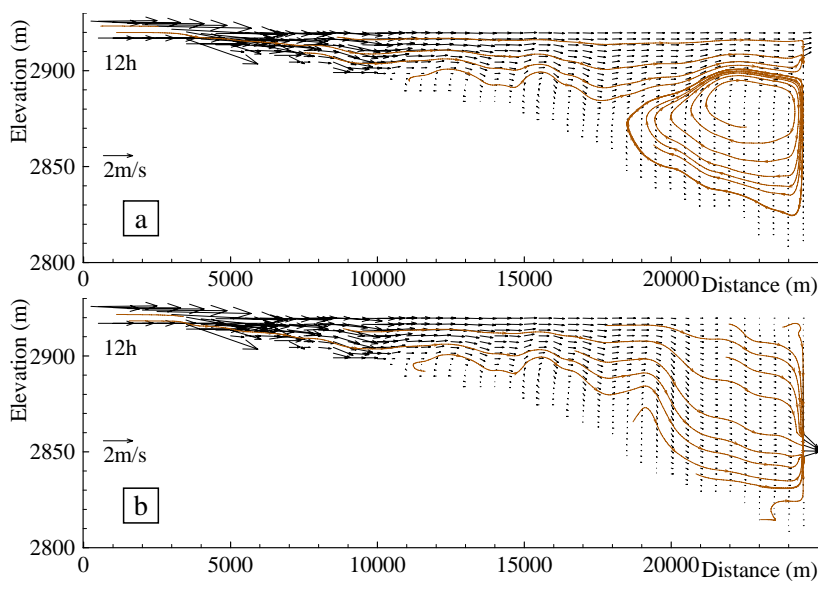

Fig. 6. Velocity vectors and streamlines in Bala Reservoir ((a) Case 1 and Case 2; (b) Case 3).

The saturation concentration at local atmospheric pressure $C_{\mathrm{s}}$ is set at $100 \%$ in the simulation.

According to the parameter calibration in the Dachaoshan Reservoir, the dissipation coefficient, $k_{T}$, is determined to be $0.003 \mathrm{~h}^{-1}$ when the water is supersaturated. Otherwise, $k_{T}$ is assumed to be zero.

The surface mass transfer coefficient across the air-water free surface $\left(k_{\mathrm{L}} a\right)_{\mathrm{S}}$ is calculated by Eq. (10), where the wind speed is assumed to be $0.9 \mathrm{~m} \mathrm{~s}^{-1}$ according to meteorological data from the Maerkang weather station, 70 kilometers away from the Bala Dam site.

\section{Hydrodynamic and TDG Results of different operational regulations}

\subsection{Flow and temperature field}

The predicted flow patterns in the Bala Reservoir are shown in Fig. 6 with streamlines colored by vector. The discrepancies in the three cases are mainly due to the outlet elevation. Case 1 and Case 2 have a similar streamline with a large circle backflow under the main current, which is in contrast to Case 3. With respect to Case 3, the main current dives directly to the bottom at the point $7 \mathrm{~km}$ upstream of Bala Dam without obvious backflow in the field. A low velocity zone forms in the surface layers upstream of the dam. The maximum velocities of the three cases are approximately $3 \mathrm{~m} \mathrm{~s}^{-1}$ at the entrance of the reservoir. The velocity decreases further downstream because of the water depth increase. The velocity in the regions close to the Bala Dam is approximately $0.2 \mathrm{~m} \mathrm{~s}^{-1}$ for all three cases.

The initial temperature field (Fig. 3) demonstrates that the stratification of water temperature forms in Bala Reservoir in August. A $10 \mathrm{~m}$-thick transition layer lies at a $30 \mathrm{~m}$ depth beneath the water surface. The temperature decrement between the two regions above and below the transition layer is as

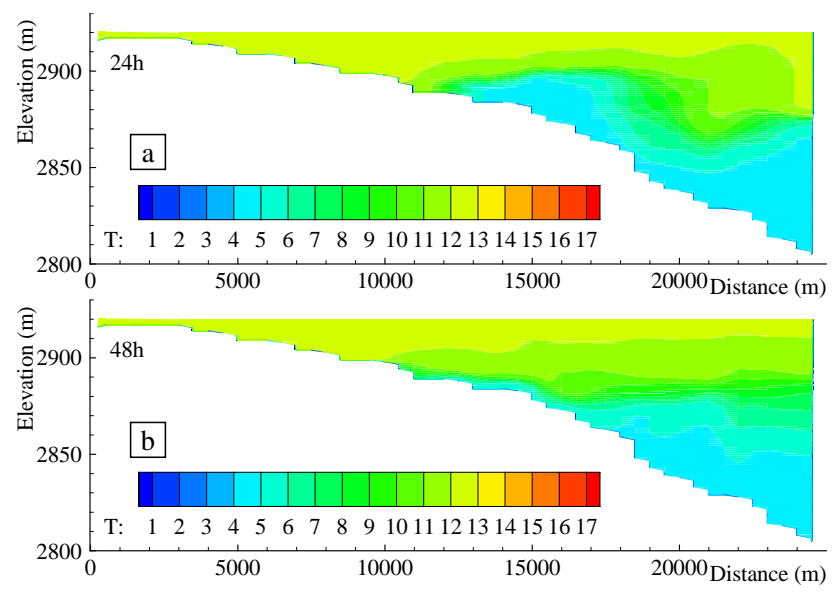

Fig. 7. Water temperature stratification in Bala Reservoir $\left(^{\circ}\right)$.

high as $8^{\circ}$. When Xiaerga starts to spill, the initial stratification breaks because of the flood flow disturbance (Fig. 7a). After the spilling gates are closed, the reservoir water is gradually pacified and a new stratification forms again; this is presented in Fig. 7b. The flood significantly impacts the temperature stratification in the reservoir.

\subsection{TDG distribution}

\subsubsection{Case 1 (continuous discharge + outflow through the high-elevation tunnel)}

In Case 1, Xiaerga adopts a continuous discharge pattern as the inflow condition and the surface spilling tunnel is chosen as the outflow passageway. The TDG distributions in Bala Reservoir at different times are demonstrated in Fig. 8.

According to Fig. 8, Xiaerga starts continuous flood discharge at the $6 \mathrm{~h}$ moment, continues for $12 \mathrm{~h}$ and the generated TDG is $141 \%$ in saturation. The contours in Fig. 8 demonstrate that the supersaturated TDG appears in the entrance of Bala Reservoir at the $6 \mathrm{~h}$ moment. The transportation of TDG is in accordance with the main directional flow. A high TDG cluster is formed at the top layers of the reservoir. At the $18 \mathrm{~h}$ moment, the effect of TDG supersaturation has already expanded to the dam with the flow current, with the maximum TDG saturation of $140.7 \%$. In contrast to the inflow TDG saturation, $141 \%$, the dissipation of TDG is slight. This is because the flood discharge transports the elevated TDG flow at a high velocity. Therefore, the resident time for TDG flow in the reservoir is not long enough to complete the TDG degassing. After $18 \mathrm{~h}$, the flood discharge is shut and the inflow is composed only of the tailrace of $\mathrm{Xi}$ aerga with a TDG saturation of $100 \%$. The supersaturated TDG flow then runs downstream of Bala at a slow pace and the degassing is sufficient because the resident time increases with the decreasing velocity. Consequently, the high TDG cluster becomes narrower and shorter over time. At the final 

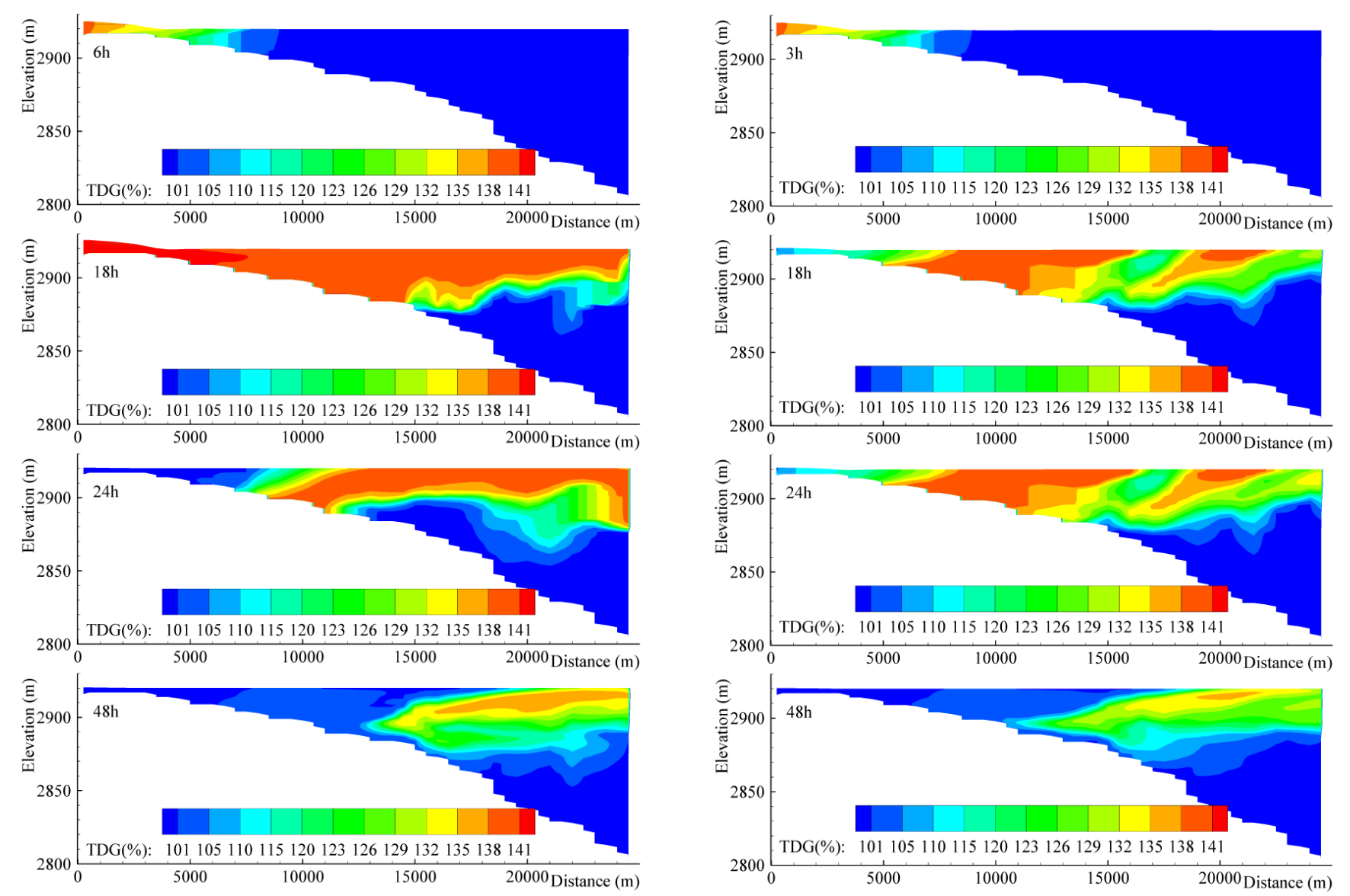

Fig. 8. The TDG distributions of Case 1 at different moments.

moment of the simulation, the high TDG cluster is $10 \mathrm{~km}$, with a maximum value of $136.2 \%$.

\subsubsection{Case 2 (interrupted discharge and outflow through the high-elevation tunnel)}

In Case 2, Xiaerga adopts an interval discharge pattern as the inflow condition and the surface spilling tunnel is chosen as the outflow passageway. The TDG distributions in Bala Reservoir at different times are presented in Fig. 9.

At the $3 \mathrm{~h}$ moment, the spillway of Xiaer begins to work. The spillway gate is alternately opened and closed four times every $3 \mathrm{~h}$. Similar to Case 1, the high TDG clusters still appear at the top layers of Bala Reservoir as a result of the TDG transporting with the flow current. In contrast to Case 1, several clusters appear in the reservoir concurrently because of the interval flood discharge pattern. Figure 9 demonstrates that at the $18 \mathrm{~h}$ moment, four times the flood discharge brings the TDG cluster to the dam. At the $24 \mathrm{~h}$ moment, the flood discharge is shut permanently in the simulation. The TDG in the reservoir then dissipates under the function of attenuation and sufficient degassing. At the end of the simulation, the negative region of TDG supersaturation is narrower than

Fig. 9. The TDG distributions of Case 2 at different moments.

that of the prior moment, with a maximum TDG saturation of $135.3 \%$.

\subsubsection{Case 3 (continuous discharge and outflow through the low-elevation tunnel)}

In Case 3, Xiaerga adopts a continuous discharge pattern as the inflow condition and the bottom discharge tunnel is chosen as the outflow passageway. The TDG distributions in Bala Reservoir at different moments are presented in Fig. 10.

At the $6 \mathrm{~h}$ moment, the continuous flood discharge begins and lasts for $12 \mathrm{~h}$. The high TDG cluster moves forward to the dam under high flood velocity without sufficient degassing for a long period. The TDG transport route is in accordance with the flow current streamline, therefore, the high TDG cluster spreads to the bottom as the bottom discharge tunnel is adopted as the flow passageway for Bala. The flood discharge is shut at $18 \mathrm{~h}$ and approximately $80 \%$ of the reservoir is at a TDG level of $140 \%$ at that moment. The TDG level in the reservoir then decreases because of the attenuation and sufficient degassing with the extended resident time. A high TDG cluster lies at the bottom of the reservoir in accordance with the main current. At the final moment of the simulation, 

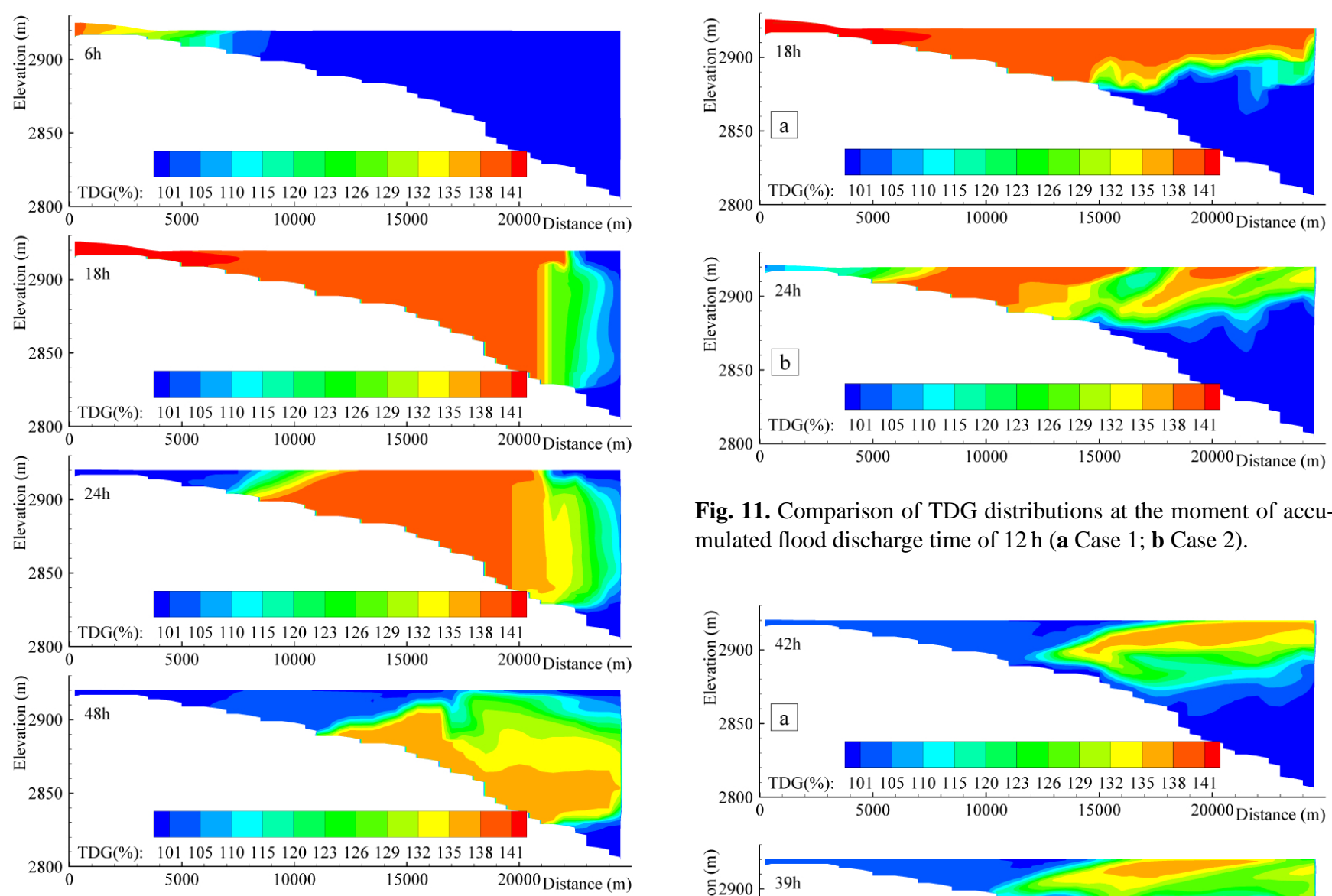

Fig. 10. The TDG distributions at the key moments (Case 3).

the negative region of TDG supersaturation is narrower, with a maximum TDG saturation of $136.8 \%$.

\section{Discussions on the effect of operational regulations}

The TDG results indicate that different inflow boundary conditions result in different distributions of TDG in Bala Reservoir. This study analyzes regulation effects from two aspects, the operation of the upstream power station (inflow condition) and the operation of the downstream power station (outflow condition).

\subsection{Regulation of the upstream power station}

Case 1 and Case 2 adopted two different inflow boundary conditions - the continuous discharge pattern and the interval discharge pattern of the upstream Xierga Dam (Fig. 4). A comparison between Case 1 and Case 2 demonstrates the effect of the regulations of Xiaerga power station, the upstream power station.

Figure 11 presents the TDG comparison of the two cases at the moment when the discharge time accumulates to $12 \mathrm{~h}$. It demonstrates that a high TDG cluster with a thickness of

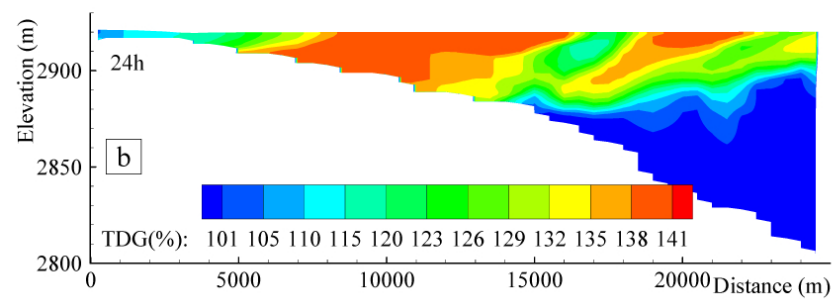

Fig. 11. Comparison of TDG distributions at the moment of accumulated flood discharge time of $12 \mathrm{~h}$ (a Case 1; b Case 2).
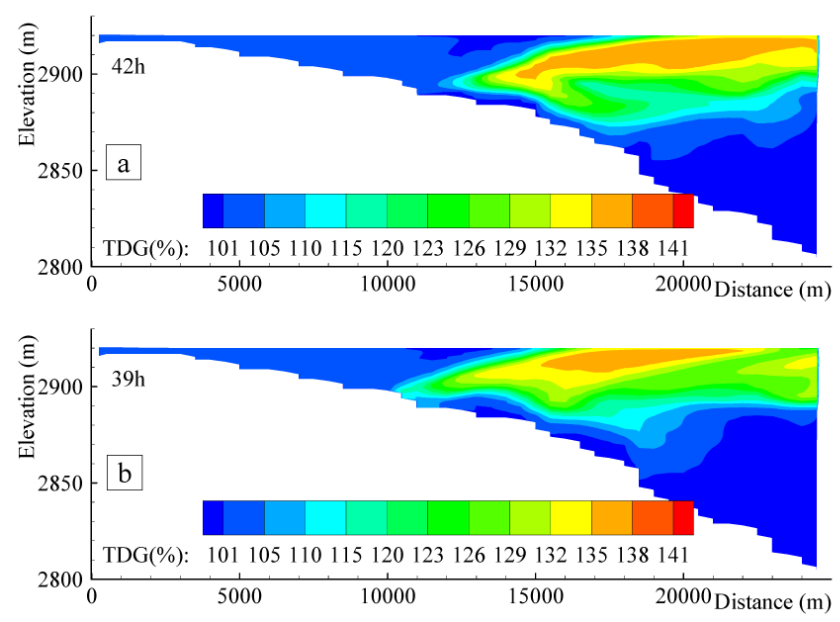

Fig. 12. Comparison of TDG distributions at the moment of accumulated off-time of $24 \mathrm{~h}$ (a Case 1; b Case 2).

$25 \mathrm{~m}$ is formed at the surface of Bala Reservoir in Case 1, with a maximum TDG saturation of over $138 \%$. Several high TDG clusters are formed in Case 2 that are caused by the interval flood discharge pattern. The high-TDG area in Case 2 is significantly smaller than that of Case 1 .

A comparison of the two cases at the moment when the off-time of the spillway tunnel accumulates to $24 \mathrm{~h}$ is presented in Fig. 12. The contrast demonstrates that the TDG levels are significantly less than at the same moment in Fig. 11. For both cases, the high TDG clusters are distributed around the surface layers in the $13 \mathrm{~km}$ range in the front of Bala Dam.

To quantitatively compare the supersaturated TDG effect of the two cases, accumulative curves of area ratio are drawn in Fig. 13. The horizontal coordinate represents the TDG percent saturation. The vertical coordinate is defined as the area ratio of the TDG saturation area that is higher than the 


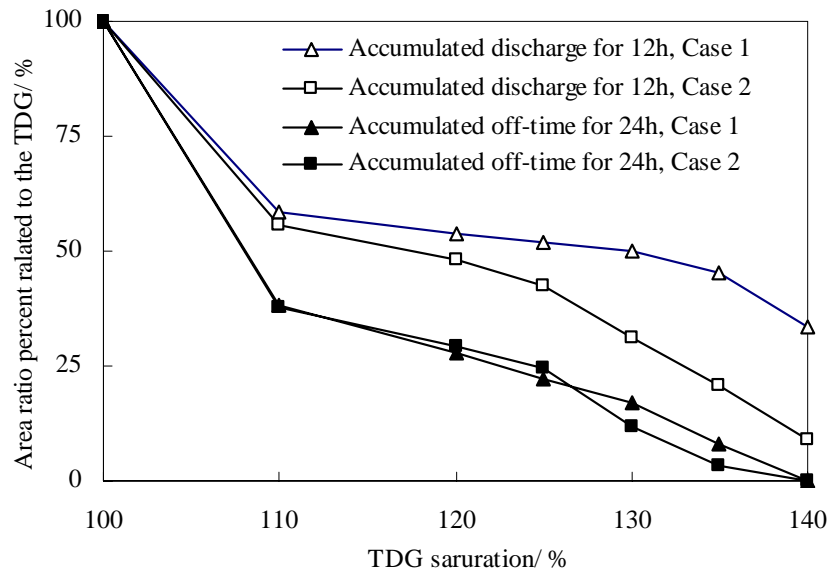

Fig. 13. Accumulated curves of area ratio percent.
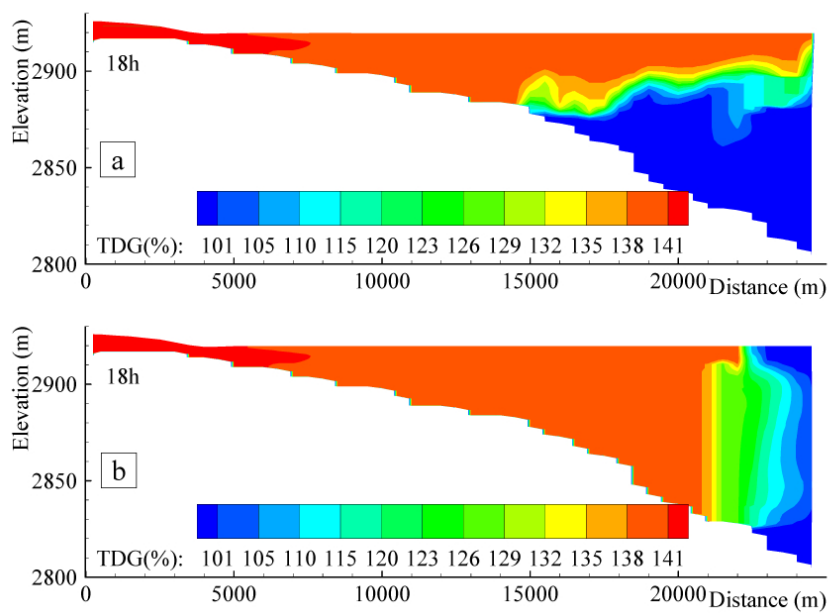

Fig. 14. Comparison of TDG distribution of Case 1 (a) and Case 3 (b) at the moment of accumulative discharge time of $12 \mathrm{~h}$.

corresponding saturation for the whole reservoir. At the moment when the flood discharge time accumulates to $12 \mathrm{~h}$, the area ratio percent of Case 2 is much lower than Case 1. When the off-time accumulates to $24 \mathrm{~h}$, the area ratio percent of the two cases is proximal, with the exception of TDG, which ranges from 130 to $135 \%$.

To minimize the TDG supersaturation in a reservoir, the comparison of Case 1 and Case 2 demonstrate that an upstream interval-discharge pattern is more effective than a continuous flood discharge pattern.

\subsection{Regulation of downstream power stations}

Case 1 and Case 3 adopt different release structures of the downstream Bala Dam, which are operated with different elevations. The surface spillway tunnel at $2903 \mathrm{~m}$ is chosen in Case 1 and the bottom discharge tunnel at $2845 \mathrm{~m}$ is chosen for Case 3. The inflow boundaries are the same for the two cases. The comparison between Case 1 and Case 3 demon-
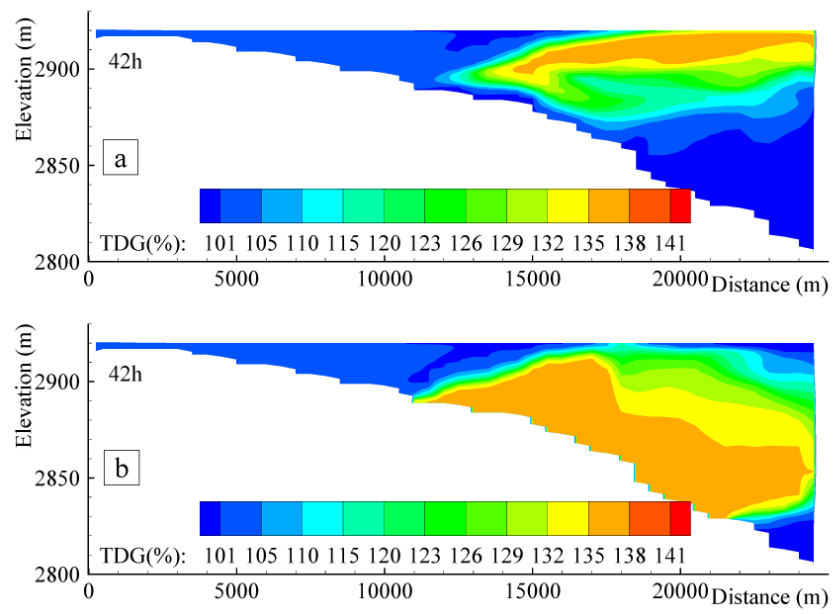

Fig. 15. Comparison of TDG distribution of Case 1 (a) and Case 3 (b) at the moment of accumulated off-time of $24 \mathrm{~h}$.

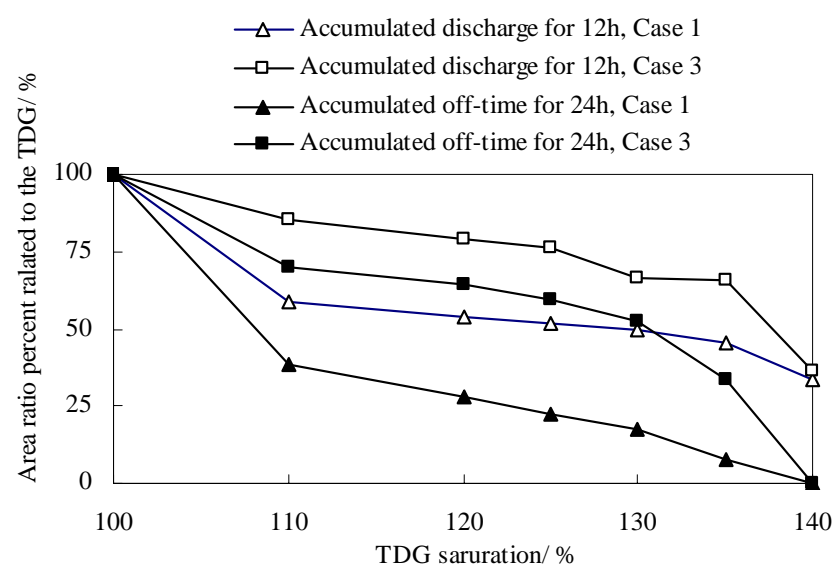

Fig. 16. Accumulated area ratio over TDG saturation level.

strate the effect of operational regulations on the Bala power station, the downstream power station.

Figure 14 presents the TDG comparison of the two cases at the moment when the discharge time accumulates to $12 \mathrm{~h}$. It demonstrates that a high TDG cluster with a thickness of $25 \mathrm{~m}$ is formed at the surface of Bala Reservoir in Case 1. In Case 3, the supersaturated TDG spread from the surface to the bottom as the surface streamlines turns towards the bottom. The high-TDG area in Case 3 is significantly larger than that of Case 1.

Another comparison of the two cases is analyzed at the moment when the off-time of the spillway tunnel accumulates to $24 \mathrm{~h}$ and is presented in Fig. 15. The contrast demonstrates that the TDG levels are significantly decreased than at the time in Fig. 14. In Case 1, the high TDG cluster is distributed around the surface layers in the $13 \mathrm{~km}$ range in front of Bala Dam. In Case 3, the high TDG cluster is distributed at the bottom $15 \mathrm{~km}$ range at the front of the dam. 
To minimize the TDG supersaturation in a reservoir, the comparison of Case 1 and Case 3 demonstrate that a downstream surface-discharge pattern is more effective than a bottom-discharge pattern.

\section{Conclusions}

The regulation of operations is an effective way to mitigate the harmful TDG supersaturation in the Bala Reservoir on the Zumuzu River. This paper employed a laterally averaged two-dimensional model to simulate the TDG distributions of different spill scenarios, including the configuration combinations of the upstream and downstream power stations.

The results indicate that the TDG distribution is mainly affected by the movement of the current flow. When a spillway is discharged, the supersaturated TDG moves rapidly towards the Bala Dam with the main current. Small amounts of dissipation happen in such a deep reservoir. The TDG saturation level remains high until it reaches the downstream Bala Dam at a maximum TDG level that is higher than $138 \%$. The protection of aquatic organisms in the large reservoirs during the discharging period is a concern.

According to the comparison of the scenario simulation results, the optimized regulations for the reservoir are concluded. For the upstream power station, an interval-discharge pattern instead of a continuous-discharge pattern is recommended to minimize the negative effect in the reservoir that is caused by supersaturated TDG. For the downstream power station, the use of a surface tunnel rather than a bottom tunnel is recommended as a release structure.

Future modeling efforts are suggested to combine the endurance of fish with respect to supersaturated TDG and to propose mitigation measures. The effect of the regulation on the downstream river is also another important and sophisticated problem that needs to be addressed respectively in the future.

Acknowledgements. This material is based upon work supported by the National Natural Science Foundation of China, Grant No. 51179111.

Edited by: Y. Cai

\section{References}

Bentley, W. W., Dawley, E. M., and Newcomb, T. W.: Some effects of excess dissolved gas on squawfish, Ptychocheilus oregonensis (Richardson), Technical Information Center, Oak Ridge, Tennessee, 41-46, 1976.

Cai, Y. P., Huang, G. H., Yang, Z. F., and Tan, Q.: Identification of optimal strategies for energy management systems planning under multiple uncertainties, Appl. Energ., 86, 480-495, 2009.
Cai, Y. P., Huang, G. H., Tan, Q., and Chen, B.: Identification of optimal strategies for improving eco-resilience to floods in ecologically vulnerable regions of a wetland, Ecol. Modell., 222, 360-369, 2010.

Chen, S. C., Liu, X. Q., Jiang, W., and Li, K. F.: Effects of total dissolved gas supersaturated water on lethality and catalase activity of Chinese Sucker (Myxocyprinus asiaticus Bleeker), J. Zhejiang University-SCIENCE B (Biomedicine \& Biotechnology), 13, 791-796, 2012.

Feng, J. J., Li, R., Li, K. F., and Li, J.: Study on release process of supersaturated total dissolved gas downstream of high dam, J. Hydroelec. Eng., 29, 7-12, 2010 (in Chinese).

Feng, J. J., Li, R., Yang, H. X., and Li, J.: A laterally averaged two-dimensional simulation of unsteady supersaturated total dissolved gas in deep reservoir, J. Hydrodynam., 25, 396-403, 2013.

Fu, X. L., Li, D., and Zhang, X. F.: Simulations of the threedimensional total dissolved gas saturation downstream of spillways under unsteady conditions, J. Hydrodynam., 22, 598-604, 2010.

Huang, X., Li, K. F., Du, J., and Li, R.: Effects of gas supersaturation on lethality and avoidance responses in juvenile rock carp (Procypris rabaudi Tchang), J. Zhejiang University-SCIENCE B (Biomedicine \& Biotechnology), 11, 806-811, 2010.

Lauri, H., de Moel, H., Ward, P. J., Räsänen, T. A., Keskinen, M., and Kummu, M.: Future changes in Mekong River hydrology: impact of climate change and reservoir operation on discharge, Hydrol. Earth Syst. Sci., 16, 4603-4619, doi:10.5194/hess-164603-2012, 2012.

Li, R., Li, J., Li K. F., Deng, Y., and Feng, J. J.: Prediction for supersaturated total dissolved gas in high-dam hydropower projects, Sci. China, Series E: Technological Sciences, 52, 3661-3667, 2009.

Li, R., Hodges, B. R., Feng, J. J., and Yong, X. D.: A comparison of supersaturated total dissolved gas dissipation with dissolved oxygen dissipation and reaeration, J. Environ. Eng. ASCE, 139, 385-390, 2013.

Liang, R. F., Li, B., Li, K. F., and Tuo, Y. C.: Effect of total dissolved gas supersaturated water on early life of David's schizothoracin (Schizothorax davidi), J. Zhejiang University-SCIENCE B (Biomedicine \& Biotechnology), 14, 632-639, 2013.

Lindim, C., Pinho, J. L., and Vieira, J. M. P.: Analysis of spatial and temporal patterns in a large reservoir using water quality and hydrodynamic modeling, Ecol. Modell., 222, 2485-2494, 2011.

Liu, X. Q., Li, K. F., Du, J., Li, J., and Li, R.: Growth rate, catalase and superoxide dismutas activities in rock carp (Procypris rabaudi Tchang) exposed to supersaturated total dissolved gas, J. Zhejiang University-SCIENCE B (Biomedicine \& Biotechnology), 12, 909-914, 2011.

Ma, Q., Li, R., Feng, J. J., and Wang, L. L.: Relationships between total dissolved gas and dissolved oxygen in water, Fresenius Environ. Bull., 22, 3243-3250, 2013.

Nikoo, M. R., Karimi, A., and Kerachian, R.: Optimal Long-term Operation of Reservoir-river Systems under Hydrologic Uncertainties: Application of Interval Programming, Water Resour. Manage., 27, 3865-3883, 2013.

O'Connor, D: Wind effects on gas-liquid transfer coefficients, J. Environ. Eng.-ASCE, 109, 731-752, 1983. 
Politano, M., Carrica, P., and Weber, L.: A multiphase model for the hydrodynamics and total dissolved gas in tailrace, Int. J. Multiphase Flow, 35, 1036-1050, 2009.

Politano, M., Amado, A. A., Bickford, S., and Hay, D.: Evaluation of operational strategies to minimize gas supersaturation downstream of a dam, Comput. Fluids, 68, 168-185, 2012.

Qu, L., Li, R., Li, J., Li, K. F., and Deng, Y.: Field observation of total dissolved gas supersaturation of high-dams, Sci. China, Series E: Technological Sciences, 54, 156-162, 2011.

Rucker, R. R.: Gas-bubble disease: Moralities of coho salmon, Oncorhynchus kisutch, in water with constant total gas pressure and different oxygen-nitrogen ratio, Fish Bull., 73, 915-918, 1976.

Urban, A. L., Gulliver, J. S., and Johnson, D. W.: Modeling total dissolved gas concentration downstream of spillways, J. Hydraul. Eng.-ASCE, 134, 550-561, 2008.

van Vliet, M. T. H., Yearsley, J. R., Franssen, W. H. P., Ludwig, F., Haddeland, I., Lettenmaier, D. P., and Kabat, P.: Coupled daily streamflow and water temperature modelling in large river basins, Hydrol. Earth Syst. Sci., 16, 4303-4321, doi:10.5194/hess-16-4303-2012, 2012.
Weitkamp, D. E., Sullivan, R. D., Swant, T., Zhao, Y. W., and Chen, H.: Gas bubble disease in resident fish of the lower Clark Fork River, Trans. Am. Fisheries Soc., 132, 865-876, 2003.

Yang, Z. J., Liu, D. F., and Ji, D. B.: An eco-environmental friendly operation: An effective method to mitigate the harmful blooms in the tributary bays of Three Gorges Reservoir, Sci. China Technol. Sci., 56, 1458-1470, 2013.

Yi, Y. J., Wang, Z. Y., and Yang, Z. F.: Impact of the Gezhouba and Three Gorges Dams on habitat suitability of carps in the Yangtze River, J. Hydrol., 387, 283-291, 2010.

Yin, X., Yang, Z. F., Yang, W., and DosSantos, J.: Optimized reservoir operation to balance human and riverine ecosystem needs: model development, and a case study for the Tanghe reservoir, Tang river basin, China, Hydrol. Process., 24, 461-471, 2010. 